\title{
New drugs: T-score for transparency
}

Access to information about drugs is essential for the quality use of medicines. Pharmaceutical companies and regulatory agencies, such as the Therapeutic Goods Administration (TGA), hold large quantities of information about individual drugs, but do not always share this information. To encourage transparency, Australian Prescriber rates companies' willingness to provide clinical information about new drugs. Table 1 shows how the companies have performed between January 2009 and December 2010.

The TGA is now publishing Australian Public Assessment Reports (AusPARs) for prescription medicines. While the Editorial Executive Committee welcomes this move to greater transparency, it will still ask companies to provide the clinical evaluations for their new products. While there are similarities, the AusPAR may not include all the details found in the regulator's clinical evaluation.
For 2011, the T-score has been revised to include the AusPAR.

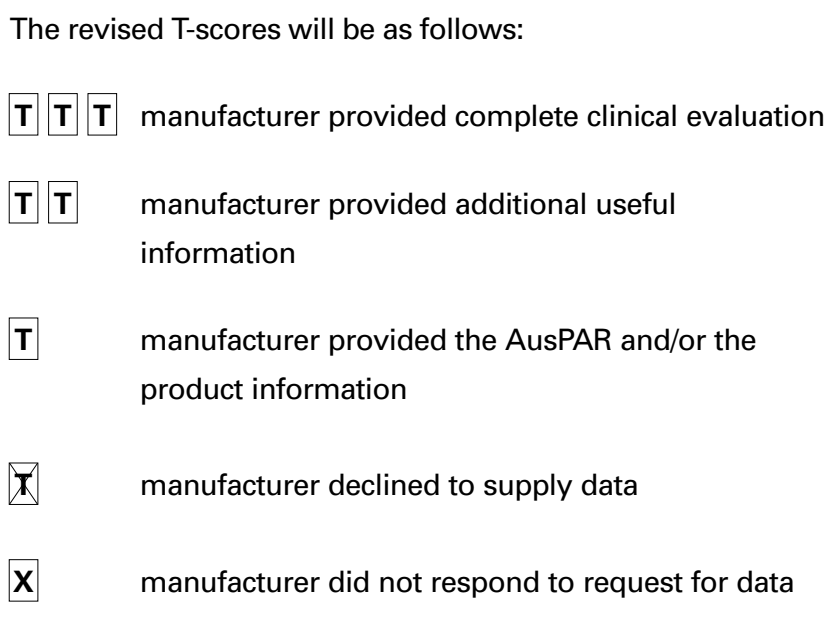

Table 1

Pharmaceutical company responses to requests for clinical evaluation data for drugs marketed Jan 2009 - Dec 2010

Company Drug

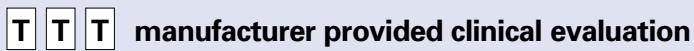

$\begin{array}{ll}\text { Amgen } & \begin{array}{l}\text { denosumab } \\ \text { degarelix } \\ \text { Pfizer }\end{array} \\ \text { anidulafungin } \\ \text { eletriptan } \\ \text { cilostazol } \\ \text { PharmaLink } & \text { caffeine citrate } \\ \text { Roche } & \text { methoxy polyethylene glycol-epoetin beta } \\ & \text { tocilizumab } \\ \text { Sanofi Pasteur } & \text { H5N1 influenza vaccine } \\ \text { Shire } & \text { icatibant } \\ \text { Wyeth } & \text { methylnaltrexone }\end{array}$

$\mathbf{T} \mathbf{T}$ manufacturer provided additional useful information

$\begin{array}{ll}\text { Abbott } & \text { omega-3-acid ethyl esters } \\ \text { Celgene } & \text { azacitidine } \\ \text { Commercial Eyes } & \text { melatonin } \\ \text { CSL } & \text { H1N1 influenza vaccine } \\ & \text { Japanese encephalitis vaccine } \\ \text { Eli Lilly } & \text { prasugrel }\end{array}$


...Table continued

\begin{tabular}{|c|c|}
\hline Company & Drug \\
\hline Genzyme & plerixafor \\
\hline GlaxoSmithKline & pneumococcal polysaccharide conjugate vaccine \\
\hline Merck Sharp \& Dohme & rizatriptan \\
\hline Orphan & nitisinone \\
\hline Sanofi-Aventis & alfuzosin \\
\hline Servier & agomelatine \\
\hline UCB Pharma & certolizumab pegol \\
\hline Wyeth & desvenlafaxine succinate \\
\hline \multicolumn{2}{|c|}{$\mathbf{T}$ manufacturer provided only the product information } \\
\hline Actelion & miglustat \\
\hline Baxter Healthcare & vaccinia smallpox vaccine \\
\hline Bayer Schering & rivaroxaban \\
\hline CSL & nebivolol \\
\hline \multirow[t]{2}{*}{ GlaxoSmithKline } & dutasteride \\
\hline & pazopanib \\
\hline Hospira & clofarabine \\
\hline Janssen-Cilag & doripenem \\
\hline \multirow[t]{2}{*}{ Merck Sharp \& Dohme } & etoricoxib \\
\hline & sugammadex \\
\hline \multirow[t]{2}{*}{ Novartis } & valsartan, amlodipine/valsartan, valsartan/hydrochlorothiazide \\
\hline & daptomycin \\
\hline Phebra & arsenic trioxide \\
\hline Sanofi Pasteur & influenza seasonal vaccine \\
\hline UCB Pharma & lacosamide \\
\hline \multicolumn{2}{|c|}{ IT manufacturer declined to supply data } \\
\hline Boehringer Ingelheim & dabigatran etexilate \\
\hline CSL & H5N1 influenza vaccine \\
\hline CSL Bioplasma & human $\mathrm{C} 1$ esterase inhibitor \\
\hline Ipsen & triptorelin embonate \\
\hline Janssen-Cilag & etravirine \\
\hline & ustekinumab \\
\hline Merck Sharp \& Dohme & golimumab \\
\hline Novartis & indacaterol \\
\hline & vildagliptin \\
\hline \multicolumn{2}{|c|}{$\mathrm{X}$ manufacturer did not respond to request for data } \\
\hline Alexion Pharmaceuticals & eculizumab \\
\hline \multirow[t]{2}{*}{ GlaxoSmithKline } & ambrisentan \\
\hline & H5N1 influenza vaccine \\
\hline
\end{tabular}

